\title{
Defect Detection Capability of Pulsed Transient Thermography
}

\author{
by S. Quek, and D.P. Almond
}

UK Research Centre in NDE, Department of Engineering and Applied Science, University of Bath, Bath, BA2 7AY.

e-mail: D.P.Almond@bath.ac.uk

\begin{abstract}
In this work, investigations have been conducted on a novel technique for enhancing thermographic data, which is known as thermographic signal reconstruction, to assess its defect detection limitation on CFRP composite. For defect greater than $4 \mathrm{~mm}$ diameter, results have indicated a $60 \%$ improvement in detectability.
\end{abstract}

\section{Introduction}

Pulsed thermography is gaining wide acceptance in the aerospace, automotive and power generation industries, owing to its quickness of inspection, repeatability and sensitivity. One commercially available system is the ThermoScope developed by Thermal Wave Imaging Inc (TWI) in USA. It consists of a central control unit, an imaging head and a complete suite of image acquisition and processing software.

The very essence of the system is the thermographic signal reconstruction (TSR) algorithm, which allows significant enhancement of the acquired data sequence. The process yields three types of images, namely: synthetic image and first and second time derivative images. These images reveal smaller and/or deeper defects or subtle features that are undetectable in the raw images and are reported by TWI in several publications [1-4]. The objective of this work is to make a quantitative assessment of the defect detection capabilities of these imaging processes.

\section{Principles of Pulsed Thermography and TSR Technique}

During inspection, a brief and high intensity flash is applied to heat up the sample. The decay of the sample surface temperature is detected and acquired by an infrared camera. Each image in the acquired sequence corresponds to the surface temperature at a particular time. Any subsurface anomaly will give rise to a local temperature increase and therefore resulting in thermal contrast [5]. Typically, the logarithmic time histories of the pixels in the anomalous and sound regions have the profiles as depicted by figure 1 [6].

In the TSR process, a polynomial function that has the form as equation 1 is fitted to the logarithmic time history of each individual pixel in the raw data. Subsequently, the first and second time derivatives are calculated using equation 2 and 3.

$$
\begin{aligned}
& \ln T=\sum_{n=0}^{\infty} a_{n}(\ln t)^{n}=a_{0}+a_{1} \ln t+a_{2}(\ln t)^{2}+a_{3}(\ln t)^{3}+\cdots+a_{n}(\ln t)^{n} \\
& \frac{d \ln T}{d \ln t}=\sum_{n=1}^{\infty} n a_{n}(\ln t)^{n-1}=a_{1}+2 a_{2} \ln t+\cdots+n a_{n}(\ln t)^{n-1}
\end{aligned}
$$




$$
\frac{d^{2}(\ln T)}{d(\ln t)^{2}}=\sum_{n=2}^{\infty} a_{n} n(n-1)(\ln t)^{n-2}=2 a_{2}+6 a_{3} \ln t+\cdots+n(n-1) a_{n}(\ln t)^{n-2}
$$

This technique has the following advantages over many conventional approaches in processing thermographic data:

1. It retains the fidelity of the low frequency thermal event and accentuates the signal contributed by a subsurface anomaly whilst suppressing high frequency temporal noise and non-thermal events.

2. It is also an effective data compression method by which the entire acquired data sequence can be replaced and recreated using a set of polynomial coefficients. The storage space is now reduced from approximately $49 \mathrm{MB}$ down to $5 \mathrm{MB}$. This in turn facilitates large area inspection, which requires multiple image acquisitions at different spots for full coverage [8].

3. Advanced mathematical operations such as FFT and differentiation can now be performed quickly without having the undesired noise problems [8].

\section{Samples and Experimental Procedures}

The state-of-the-art Thermoscope was utilised for the experimental work. Its imaging head consists of a flash heating system (power output from lamp is $2 \mathrm{~kJ}$ in 2 to $5 \mathrm{~ms}$ ) and a medium wave infrared camera (Merlin 3-5 $\mu \mathrm{m}$ by Indigo), which uses a cooled indium antimonide detector with a frame rate of $60 \mathrm{~Hz}$ and a focal plane array pixel format of $320(\mathrm{H}) \times 256(\mathrm{~V})$.

Two sets of test-pieces containing back-drilled cylindrical hole defects at varied depths were used. The first consisted of aluminium, glass fibre reinforced plastic (GFRP) and mild steel samples and the second was a total of 4 carbon fibre reinforced plastic (CFRP) composite samples. The first provided a qualitative validation on the applicability and repeatability of the TSR technique for different engineering materials while the second allowed a quantitative assessment of its limitation on defect detection for CFRP composite.

Table 1. Relevant information for each of the composite samples

\begin{tabular}{|r|c|c|c|c|}
\hline Items & Sample 1 & Sample 2 & Sample 3 & Sample 4 \\
\hline Thickness $(\mathbf{m m})$ & 7 & 7 & 7 & 14 \\
\hline Diameter $(\mathbf{m m})$ & 2 & 4 & 6 & 10 \\
\hline Depths $(\mathbf{m m})$ & $0.25,0.5,0.75,1$ & $0.50,1.0,1.50,2$ & $2.5,2.75,3,3.25$ & $3,4.0,4.5$, \\
\hline
\end{tabular}

Each of the samples was placed beneath the imaging head and adjusted to align with the focal plane of the infrared camera. Immediately after pulsed heating the sample, image acquisition was automatically executed by the system at a preset frame rate chosen for that particular sample. It was necessary since each of the samples had distinct thermal properties and contained defect(s) with varied diameter(s) and depth(s). A frame rate of $20 \mathrm{~Hz}$ was used for the GFRP sample and $60 \mathrm{~Hz}$ for both the aluminium and mild steel samples. CFRP samples with 2, 4, 6 and $10 \mathrm{~mm}$ defects were acquired at the rate of $20,7.5,7.5$ and $3.75 \mathrm{~Hz}$. All the acquired data were further processed using MOSAIC software with the TSR technique. 


\section{Results and Discussions}

\subsection{Qualitative Assessment}

Figure 2, 3 and 4 show the raw and reconstructed images for aluminium, GFRP and mild steel samples. From the illustrated figures and by inspecting on a frame-byframe basis, the following points are noted:

- The first and second time derivative images allow detection of smaller and deeper defects that are undetectable on the raw images.

- The reconstructed (TSR) images also facilitate earlier defect detection.

\subsection{Quantitative Assessment}

Images for the CFRP sample containing $6 \mathrm{~mm}$ diameter defects are shown in figure 5. The maximum depths for detecting the 2, 4, 6 and $10 \mathrm{~mm}$ defects on the raw and TSR images are presented in figure 6 as the plots of maximum depth of detection versus defect diameter respectively. Note that the maximum depth of detection for each diameter defect was determined by viewing the movies of the raw and TSR sequences, i.e., visual inspection. The abbreviation $1^{\text {st }}$ and $2^{\text {nd }}$ used in the following denote the first and second time derivative images respectively. The plot of optimum frame rate versus defect diameter is shown in figure 7 . The following conclusions are drawn from studying the results:

- For 2, 4, 6 and $10 \mathrm{~mm}$ defects, the corresponding maximum depths of detection are $1.25,2,2.5$ and $3.35 \mathrm{~mm}$ on the raw images and are $1.75\left(1^{\mathrm{st}}\right), 3\left(1^{\mathrm{st}}\right.$ and $\left.2^{\mathrm{nd}}\right), 4\left(1^{\mathrm{st}}\right.$ and $\left.2^{\text {nd }}\right)$ and $5.34 \mathrm{~mm}\left(1^{\text {st }}\right.$ and $\left.2^{\text {nd }}\right)$ on the TSR images. This in turn results in 40 , 50,60 and $60 \%$ improvement in detectability for each diameter defect..

- For raw images, the diameter-to-depth ratio estimated for each diameter defect is 1.6, 2, 2.4 and 2.98. These ratios, except for the case of $2 \mathrm{~mm}$ defect, conform to the practical rule of thumb, which specifies the minimum size for defect detection on CFRP is that the defect diameter-to-depth ratio must be greater than 2 . Nonetheless, for reconstructed images, the diameter-to-depth ratios are 1.14, 1.33, 1.50 and 1.87 for each diameter defect and this therefore demonstrates the significant enhancement of the TSR process.

- One important finding of this experiment is that in order to detect a smaller and/or deeper defect, which is undetectable on the raw images, on the TSR images, an optimum frame rate must be used for image acquisition. Using frame rate other than the optimum frame rate will result in no improvement with the TSR images in detecting deeper defect, i.e., obtaining same results as the raw images.

- Another important observation from figure 7 is that the optimum frame rate is significantly higher for $2 \mathrm{~mm}$ defect than the others. This is the case because as compared with larger defects, the contrast of smaller defects disappear in a short period of time [7]. Therefore, a higher frame rate is required to acquire the transient phenomenon before it becomes undetectable. 


\section{Conclusion}

The studies conducted in this work have established the detection limitation of the TSR technique on CFRP. For defects greater than $4 \mathrm{~mm}$ diameter, which are common in aerospace industry, a $60 \%$ improvement in detectability is achievable with the TSR process. Nonetheless, such improvement is only possible provided that the right frame rate is applied for image acquisition in the first instance. By contrast, the improvement is less for $2 \mathrm{~mm}$ diameter defect as the need to use higher frame has resulted in a lower signal-to-noise ratio.

\section{REFERENCES}

[1] Shepard S.M., Ahmed T., Rubadeux B.A., Wang D. and Lhota J.R. - Synthetic Processing of Pulsed Thermographic Data for Inspection of Turbine Components, British Inst. of NDT, Insight, Vol. 43, No. 9, Sept 2001, p. 587-589.

[2] Shepard, S.M. - Temporal Noise Reduction, Compression and Analysis of Thermographic Image Data Sequences, US Patent 6,516,084, Feb 2003.

[3] Shepard S.M., Lhota J.R., Ahmed T. and Yu Lin Hou - Thermographic Inspection of Composite Structures, SAMPE Journal, Vol. 39, No. 5, Sep/Oct 2003.

[4] Shephard S.M., Lhota J.R., Rubadeux B.A., Wang D. and Ahmed T. Reconstruction and Enhancement of Active Thermographic Image Sequences, Optical Engineering, Vol. 42, No. 5, SPIE, May 2003, p. 1337-1342.

[5] Almond D. P. and Lau S. K. - Defect sizing by transient thermography. I. An analytical treatment, J. Phys. D: Appl. Phys. 27, 1994, p. 1063-1069.

[6] Krapez, Balegeas, Deom and Lepoutre - Early detection by stimulated infrared thermography, Advances in Signal Processing for Nondestructive Evaluation of Materials, Maldague X.P., Ed., Kluwer Academic Publishers, The Nertherland, 1994, p. 303-321.

[7] Saintey M.B. and Almond D. P. - Defect sizing by transient thermography. II. A Numerical Treatment, J. Phys. D: Appl. Phys. 28, 1995, p. 2539-2546.

[8] Shepard S.M., Lhota J.R., Hou Y. and Ahmed T. - Gold standard comparison of thermographic sequence data, British Inst. of NDT, Insight, Vol 46, No. 4, April 2004.

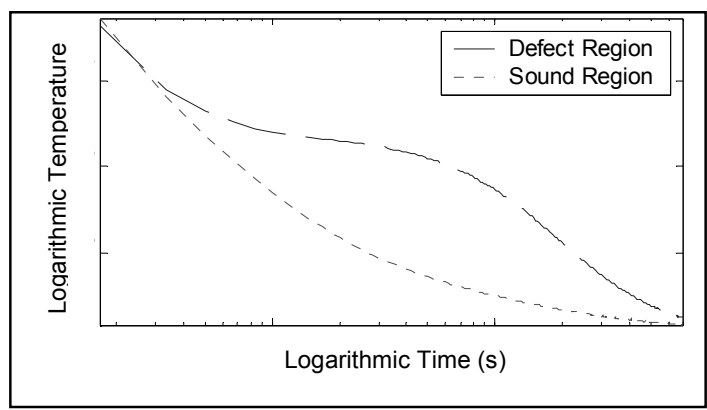

Fig. 1. Logarithmic time histories of the pixels in the anomalous and sound regions. 


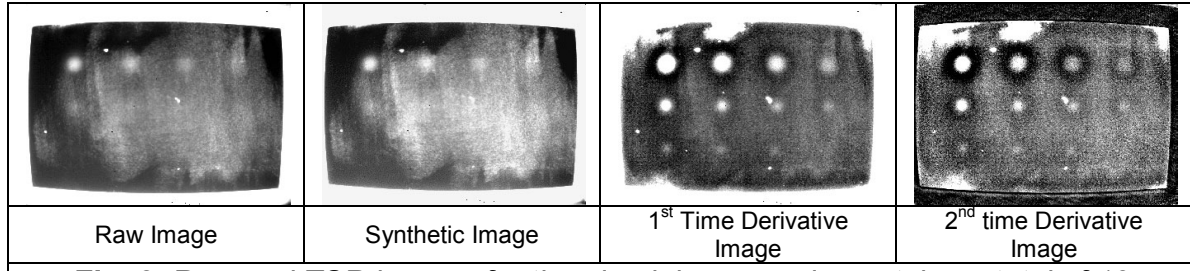

Fig. 2. Raw and TSR images for the aluminium sample contains a total of 12 defects with different diameters and depths. The $1^{\text {st }}$ and $2^{\text {nd }}$ time derivative images have revealed those that are not shown on the raw image.
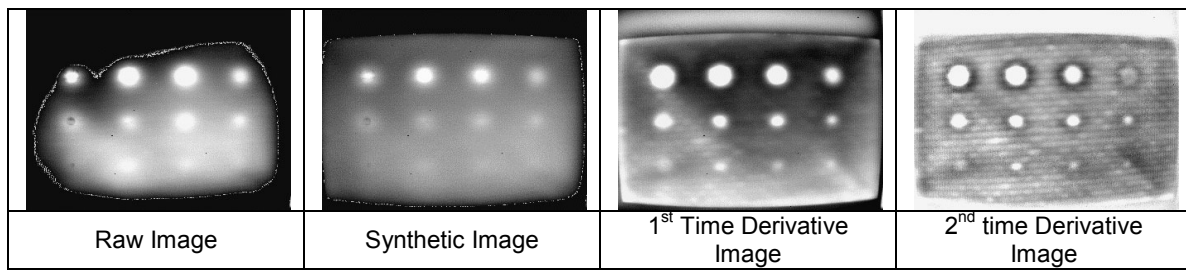

Fig. 3. Raw and TSR images for the GFRP sample contains a total of 12 defects with different diameters and depths. Defects that appear blurred on the raw image are enhanced and become visible on the $1^{\text {st }}$ and $2^{\text {nd }}$ time derivative images.

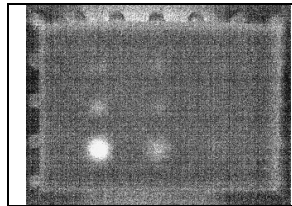

Raw Image

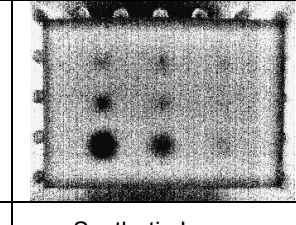

Synthetic Image

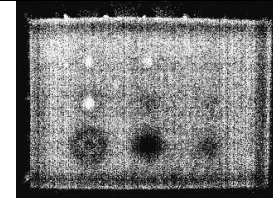

$1^{\text {st }}$ Time Derivative Image

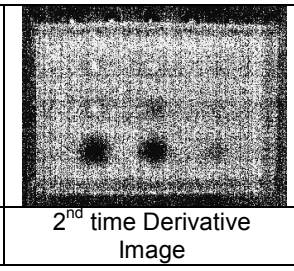
Image

Fig. 4. Raw and TSR images of mild steel sample contains a total of 9 defects with different diameters and depths. Eight of those are detected on the TSR images.

(Courtesy of TWI).

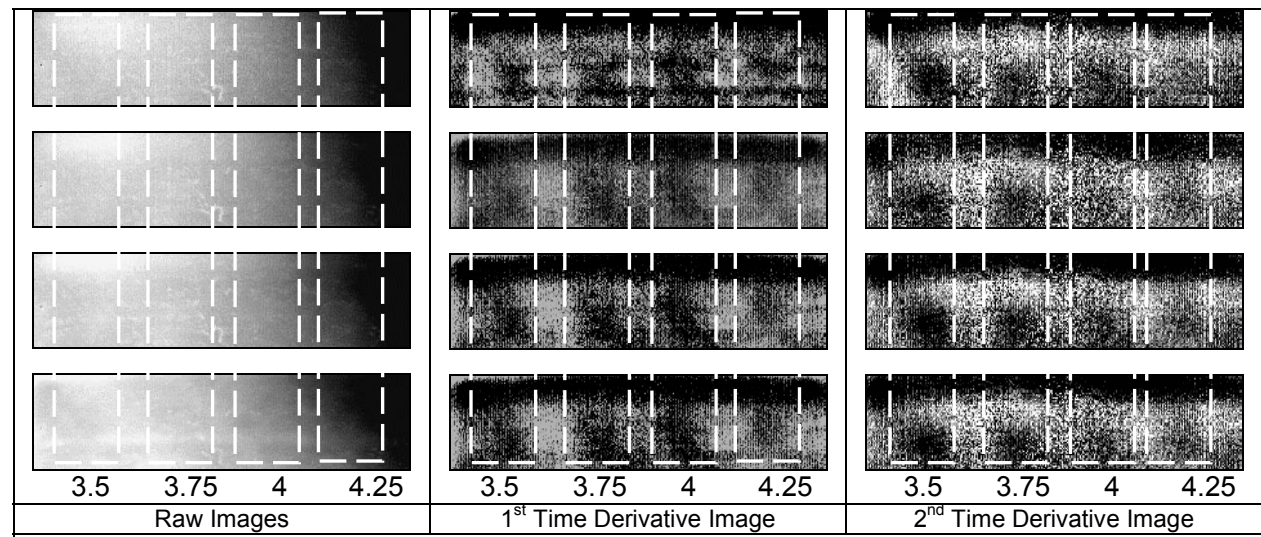

Fig. 5. The raw and TSR image sequences for the CFRP sample with $6 \mathrm{~mm}$ diameter defect at the depths of 3.5, 3.75, 4 and $4.25 \mathrm{~mm}$. These defects are not detectable on the raw image sequence but the dark spots on the $1^{\text {st }}$ and $2^{\text {nd }}$ time derivative image sequences reveal their presence. 
http://dx.doi.org/10.21611/qirt.2004.024

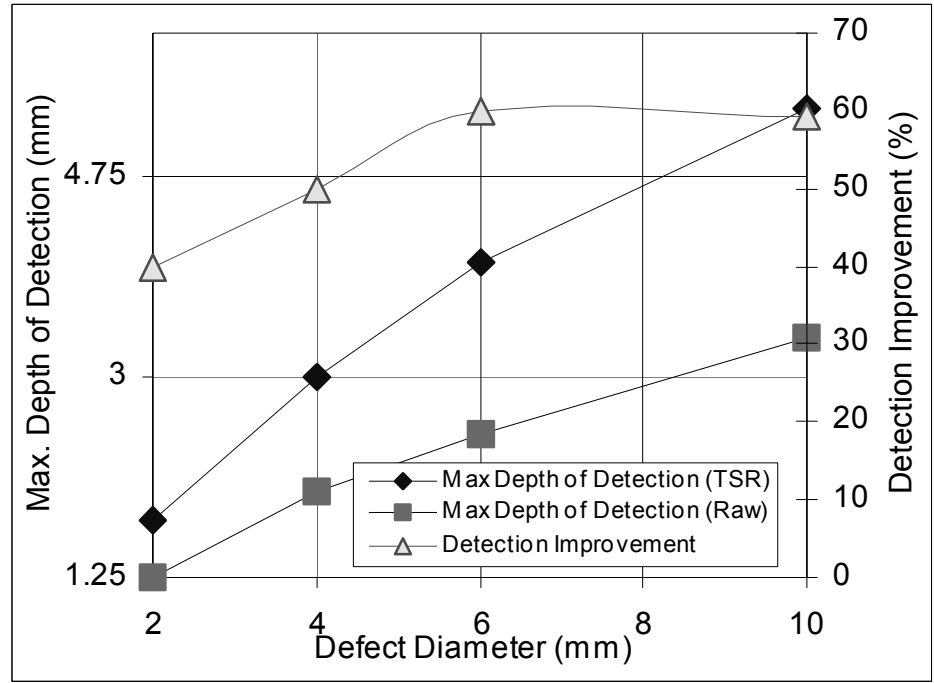

Fig. 6. The plots of maximum depth of detection versus defect diameter.

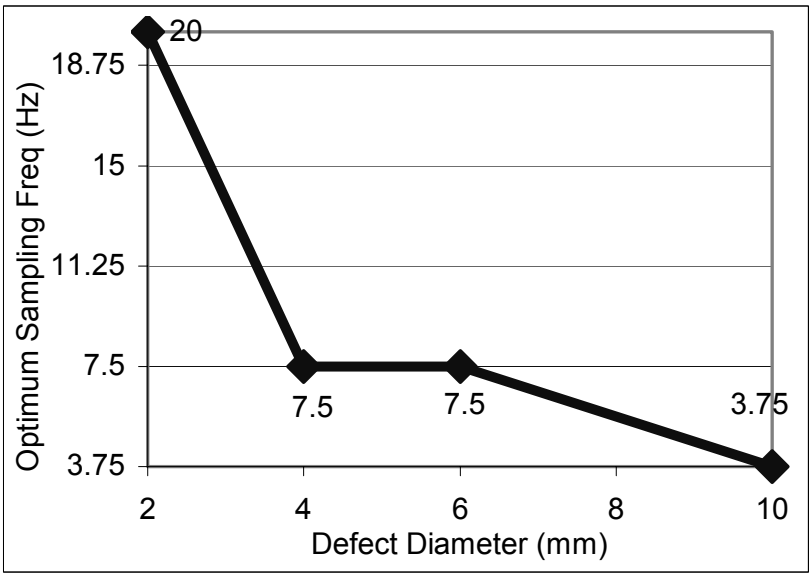

Fig. 7. The plot of optimum frame rate versus defect diameter. 